\title{
The Design and Analysis of one Kind of Internal Support Capture Mechanism for Non-cooperation Goal Satellite
}

\author{
Kailuan Sun ${ }^{1}$, Jiatai Wang ${ }^{1}$, Qi Xu ${ }^{1}$, Zhen Yang ${ }^{2}$ \\ 1. Zaozhuang Vocational College of Science and Technology, Tengzhou, China, 277500 \\ 2. School of Mechanical and Electronic Engineering, North University of China
}

Keywords: Satellite; Docking ring; capture mechanism; capture technology; ADAMS

\begin{abstract}
Designed one kind to support the type capture organization in view of non-cooperation goal satellite in, the use capture organization locking goal satellite to the docking ring, could realize smoothly to the goal satellite fast capture, and reduced time the capture organization and the goal satellite contact rigid hit. Introduced the composition and working principle of the internal support capture mechanism, the capture mechanism was established based on Pro/E $3 \mathrm{~d}$ model, capture institutions to make use of ADAMS, the dynamics analysis, analysis shows that the internal brace capture mechanism to the smooth realization of the target satellite docking ring lock and release; Capture mechanism when in contact with the target satellite collision force is small, can avoid the damage to the agencies. At the same time for later laid a foundation for design and structure optimization.
\end{abstract}

\section{Introductions}

On-orbit capture technology is a very important technology in the spaceflight field, and the capture, maintenance, recycling and reusing of the spacecraft out of operation is inseparable from the technology. On-orbit capture technology refers the technology to make arrests for space target under the manned or unmanned situation ${ }^{[1]}$. The world launches $80 \sim 130$ satellite per year on average, but there are 2 3 satellite failing to the correct orbit, and among the satellite in the correct orbit, $5 \sim 10$ are out of operation at the beginning of the life, which leads to huge economic losses ${ }^{[2]}$. The development of close catch operation for non-cooperative target satellite can be used in the auxiliary aberrance for discarded satellite and the orbital rescue for fault satellite. So the study of non-cooperative target satellite capture mechanism will have a huge impact on the development of China's space industry.

The current space capture mechanisms are mainly concentrated in the study of cooperative targets, technically, which has been applied in practice for many times, and the technology is mature, such as the "cone - bar type" and variant androgynous peripheral type capture mechanism of the Soviet union. The space capture technology for non-cooperative target most uses capture mechanism with mechanical arm type, but its structure and control system are more complex and need high costs; CX - OLEV program launched by Germany and the Netherlands space agency, with satellite engine nozzle as the interface, is at risk of that nozzle are easy to be damaged. And capture mechanisms such as fly, fly claw are in the stage of development and validation. Domestic study of non-cooperative target satellite capture mechanism is relatively small.

For the problem such as malfunction or failure of non-cooperation GEO satellite, to expect to carry out auxiliary aberrance or repair work, a internal support capture mechanism is designed. Capture agencies, within the scope of the position and Angle tolerance, can achieve rapid capture operation; In addition, the collision force to the target satellite is small in the process of capture, avoiding damaging the agencies; And its mechanical structure and control system are relatively simple and need low cost. Capture mechanism will compress target satellite docking ring through three outstretched hand claws, making the target satellite and capture mechanism become an organic whole, and then recover capture mechanism, finishing the fast capture for the target satellite. 


\section{Structural design of the internal support capture mechanism}

Technical indicators:

1) Capture target: GEO satellite weight $1000-3000 \mathrm{Kg}$;

2) Position, angle tolerance is $\pm 10 \mathrm{~mm}, \pm 1^{\circ}$;

3) System function: rapid capture, soft contact, rigid connection and lock, after locking the central axis of mechanism align with the one of docking ring;

The design diagram of internal support type capture mechanism is shown in figure 1, which is mainly composed of installation bearing component, compact components, turntable component, driving mechanism, etc. The function of each part is shown below:

1) Installation bearing components: the main base, linear guide, used for installation and location of each part.

2) Compression components: used to compress the docking ring edge of target satellite, completing the capture movement.

3) Turntable components: through the drive mechanism to drive the rotation, making compression components conduct radial movement.

4) Driving mechanism: provide power supply for the entire system.

General GEO satellites take with distal engine, therefore leaving circular hole in the center of the capture mechanism to meet that within the capture tolerance it will not impact the remote engine of target satellite. The whole internal support type capture mechanism, through the interaction and cooperative work together of various components, will complete the fast compaction of target satellite docking ring, and then release docking ring after repair work is done, completing full capture and releasing process of the target satellite.

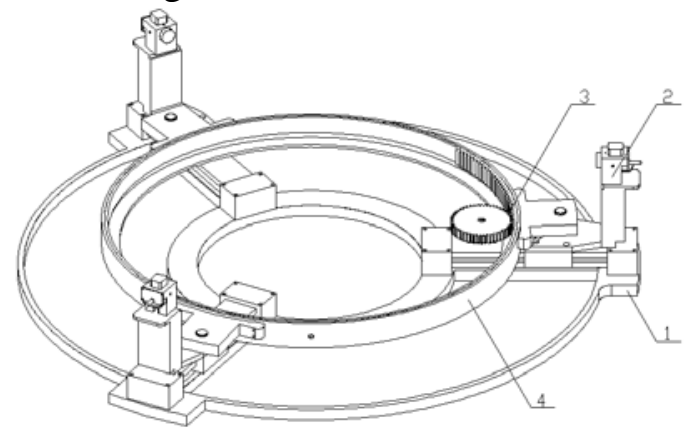

1-installation bearing component

2-compression components

3-turntable component

4-driving mechanism

Fig.1 Schematic diagram of the inner capture mechanism

\section{Operating principle of the internal support capture mechanism}

Capture principle. Service satellite adjust attitude, making it in the target satellite position and Angle tolerance range. Internal support capture mechanism moves to the target satellite direction under the drive of telescopic mechanism of service satellite, when the control components such as sensors and the CCD camera detect that a target satellite docking ring is in the capture range (pressure plate distance $8 \mathrm{~mm}$ to docking ring), motor on the capture mechanism will start, and drive rotary components, so that the pressure plate in the compression component is located above the docking ring edge, at the same time, contact plunger touches docking ring inner edge and recycles, finally makes the pressure plate impact docking ring. Telescopic institutions are drew, and the target satellite is dragged to the satellite service direction. 


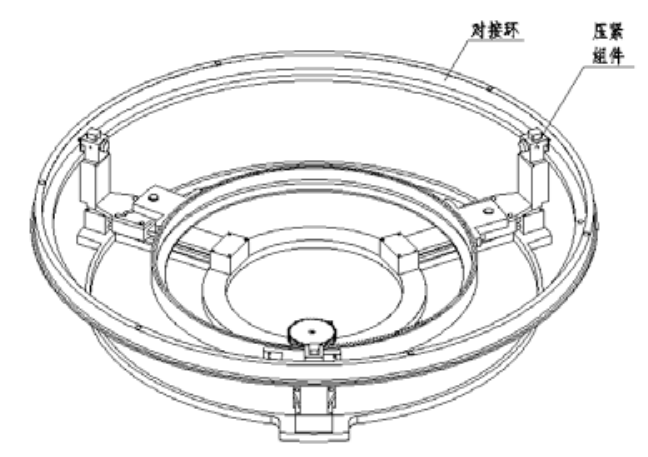

Fig.2 Schematic diagram of capture process

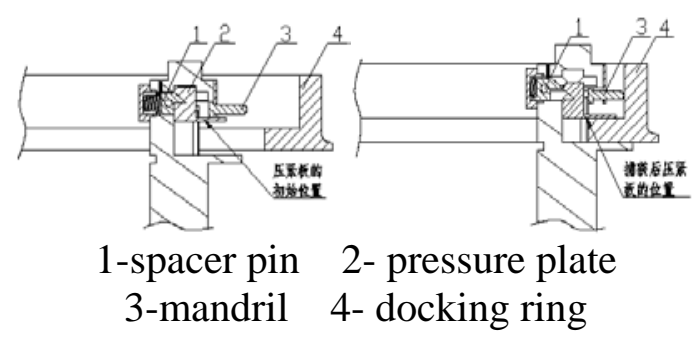

Fig.3 Captures before and after state of the clamping plate

Releasing principle. After the repair work is completed, the target satellite is needed to be released. First of all, the motor on the capture mechanism of internal brace type began to reverse, and rotary components drive compaction components to move toward the circle center, then plunger slowly recover in normal situation under the action of spring force, at the same time making the pressure plate away from the docking ring inner edge, and rotary components continue to spin, making outside diameter of capture mechanism be less than the inner diameter of the docking ring. Finally, capturing mechanism backed to the service satellite under the action of telescopic mechanism, completing the whole capture and releasing process.

\section{Dynamics analysis of the internal support capture mechanism}

Use Pro/E to set up 3D model for internal support type capture institutions and assemble, then import it into ADAMS simulation software, and make the simulation analysis capture and releasing process of capture mechanism under ideal conditions. Now make the following assumptions:

1) Before capture, the target satellite has not initial velocity;

2) If there is no special instructions, all the parts are regarded as rigid body;

3) The center shaft of capture mechanism aligns and center shaft of docking ring.

Simulation analysis of capture process. In the simulation process, the parameters of limit pin spring are set as: $\mathrm{k}=60 \mathrm{~N} / \mathrm{mm}, \mathrm{c}=0.012$, preload $100 \mathrm{~N}$. Through dynamic simulation analysis, it is concluded that the target satellite displacement curve in the capture process is shown in figure 4 . By the figure, it can be seen that in capture instants, target satellite have tiny vibration, and then implement the uniform motion slowly.

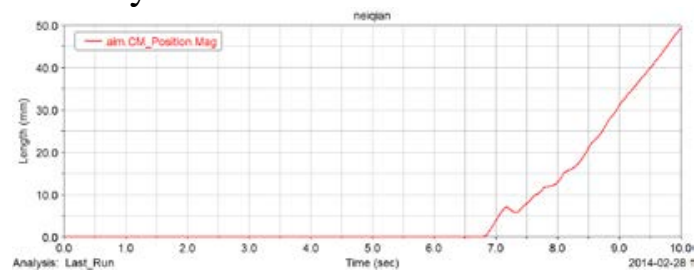

Fig.4 Displacement of the target satellite capture process

In the capture process, the plunger of the pressure component slowly contacts the inner wall of docking ring, and pushes the limit pin to move back, at the same time compressing limit pin spring. Limit pin displacement curve is shown in figure 5, and limit pin spring force curve is shown in figure 6. From figure 5 and 6 , it can be seen that the displacement of limit pin finally is almost zero, remaining stable. The figure 6 shows that maximum force of limit pin spring is about $528 \mathrm{~N}$. 


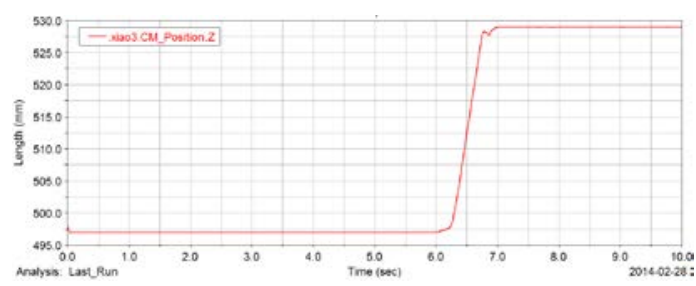

Fig.5 Displacement of pin limiting the capture process

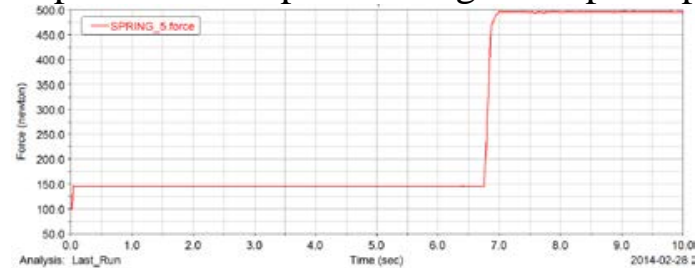

Fig.6 Spring force of pin limiting the capture process

Simulation analysis of releasing process.In the releasing process, the rotary components inverse, and the plunger of the pressure component slowly returns under the action of spring force, then limit pin also move at the same time, whose displacement curve is shown in figure 7. In the inversion process of rotary component, the limit pin spring force curve is shown in figure 8 , and the last spring force keeps around about 170N.

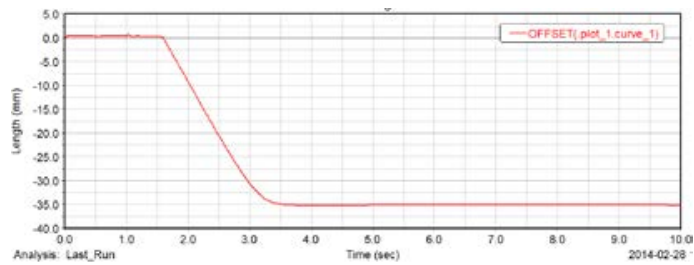

Fig.7 Displacement of pin limiting the release process

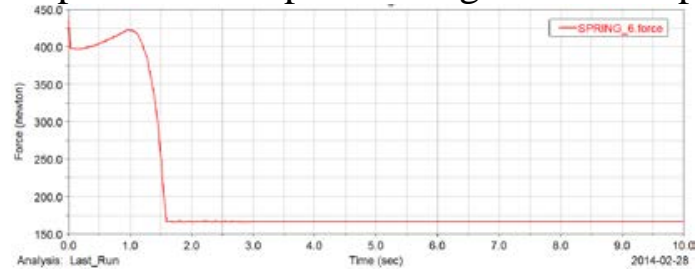

Fig.8 Spring force of pin limiting the release process

In the releasing process, capture mechanism is static relatively to the target satellite, after compact components released the docking ring, capture mechanism began to recover. The displacement curve is shown in figure 9, and it successfully completed the capture and releasing process of the target satellite.

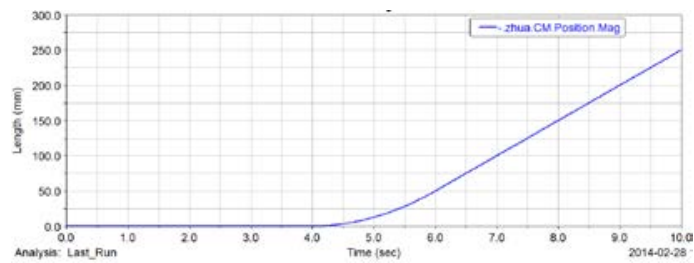

Fig.9 Displacement of the capture mechanism release process

\section{Conclusions}

Designed internal brace capture mechanism driven three compression components to make the radial move before and after by a motor, which can achieve rapid capture of non-cooperative target satellite. In addition, in the capture process, the contact force of capture mechanism to target satellite does not cause the huge shock of the target satellite.

Through the dynamic simulation analysis of capture and releasing process of internal brace type capture mechanism, it can be seen that the capture mechanism has relatively simple structure and reliable motion, which can achieve the capture of target satellite, providing a conception of the new type non-cooperative target capture mechanism for the on-orbit capture field in our country. 


\section{References}

[1] WANG Xiao-xue. Research on the docking and capturing mechanism for the uncooperative target satellites [D]. Heilongjiang: Harbin Institute of Technology,2009.

[2] LIU Chuan-shi. Study on the docking mechanism for uncooperative space target[D].HeiloJiang: Harbin Institute of Technology,2010.

[3] LI Lei,NIE Hong CHEN Jin-bao,et al. Study and design of autonomous satellite docking system[J].Machinery Design \& Manufacture,2012,11:143-145.

[4] Wenfu Xu,Bin Liang,Yangsheng Xu.Survey of modeling, Planning,and ground verification of space robotic systems [J].Acta Astronautica,2011,2.

[5] Lorenzo Tarabini,Jesus Gil,Fernando Gandia,et al.Ground guided CX-OLEV rendezvous with uncooperative geostationary satellite[J].ScienceDiret,2007,3.

[6] Xiaodong Du,Bin Liang,Wenfu Xu,et al. Pose measurement of large non-cooperative satellite based on collaborative cameras[J]. Acta Astronautica,2011(68):2047-2065.

[7] ZHANG Da-wei, TIAN Hao, ZHAO Yang,et al. Research and development of passive rocker suspension of slanetary exploration rover[J]. Journal of Astronautics,2008,29(6):1717-1721.

[8] LI Xin-gang, PEI Sheng-wei. On-orbit capture technology of spacecraft[J]. Spacecraft Engineering,2013,22(1):113-119.

[9] CAI Hong-liang,GAO Yong-ming, BING Qi-jun,et al. The research status and key technology analysis of foreign non-cooperative target in space capture system[J]. Journal of the Academy of Equipment Command \& Technology,2010,21(6):71-77.

[10]ZHAI Guang, ZHANG Jing-rui, ZHOU Zhi-cheng. A review of on- orbit life-time extension technologies for GEO satell Ites[J]. Journal of Astronautics,2012,33(7):849-859. 